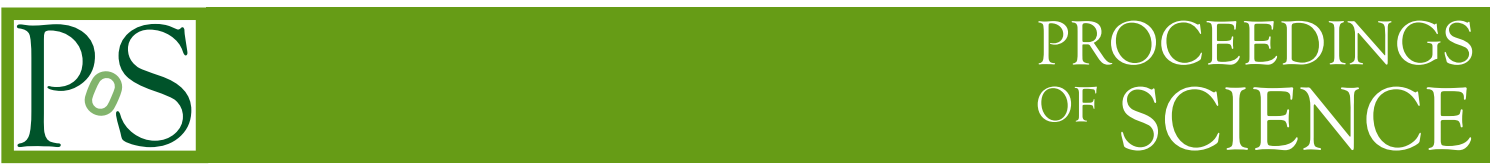

\title{
Charmonium Production at ATLAS
}

\section{S. Cheatham* on behalf of the ATLAS Collaboration. McGill University \\ E-mail: susan. cheathamecern.ch}

\begin{abstract}
Understanding of the production of P-wave charmonium states is a significant bottleneck in the understanding of charmonium production as a whole. Current theoretical calculations are limited in their ability to model the production and decay of these states. We present new ATLAS measurements of the differential production cross-sections of the $\chi_{c 1}$ and $\chi_{c 2}$ spin charmonium states, extending upon previous measurements in precision, kinematic reach, and number of measurements. The relative production cross-sections of the $\chi_{c}$ states through prompt and non-prompt production modes are explored as a function of transverse momentum, as well as the fraction of the total $\mathrm{J} / \psi$ production which occurs via $\chi_{c}$ feed-down. Prompt and non-prompt production cross-sections for $\psi(2 S)$ are presented, in the decay mode $J / \psi \pi^{+} \pi^{-}$, to help provide an accurate picture of charmonium production. Results are compared with latest theoretical predictions from a variety of theoretical approaches.
\end{abstract}

XXII. International Workshop on Deep-Inelastic Scattering and Related Subjects, 28 April - 2 May 2014

Warsaw, Poland

\footnotetext{
*Speaker.
} 


\section{Introduction.}

Study of heavy quarkonia probes hadron configuration and the non perturbative behaviour of QCD. Understanding of the production of P-wave charmonium states is a significant bottleneck in the understanding of charmonium production as a whole. Current theoretical calculations are limited in their ability to model the production and decay of these states. The Colour Singlet (CS) mechanism alone is insufficient to account for experimental measurements.

The large cross-section for inclusive charmonium production and extensive data samples available at the Large Hadron Collider (LHC) will enable detailed study of theoretical models across a large range of momentum transfer. Details of the ATLAS detector may be found in [1]. The subdetectors of greatest importance to the analyses presented here are the Inner Detector and Muon Spectrometer systems.

The inclusive production rate of prompt $J / \psi$ is the most experimentally accessible charmonium production observable at the LHC. Comparisons of experimental measurements with theoretical predictions are complicated by the large feed-down contributions from $\chi_{c}$ and $\psi(2 S)$ decays. An understanding of $\chi_{c}$ production is a crucial component of any general description of charmonium production at the LHC.

\section{Measurement of the $\chi_{c 1}$ and $\chi_{c 2}$ production with $\sqrt{s}=7 \mathrm{TeV} p p$ collisions.}

The prompt and non-prompt production cross-sections for the $\chi_{c 1}$ and $\chi_{c 2}$ charmonium states are measured with the ATLAS detector using $4.5 \mathrm{fb}^{-1}$ of integrated luminosity [2]. The $\chi_{c J}(1 P)$ states (with $\mathrm{J}=0,1,2$ ) are the only triplet of P-wave states below the open-charm threshold. Relative production of $\chi_{c 1}$ and $\chi_{c 2}$ states represents a sensitive probe of the prompt charmonium production mechanism that can provide complimentary information to the study of the S-wave states.

The $\chi_{c}$ states are reconstructed through the radiative decay $\chi_{c} \rightarrow J / \psi \gamma\left(\right.$ with $\left.\mathrm{J} / \psi \rightarrow \mu^{+} \mu^{-}\right)$ where photons are reconstructed from $\gamma \rightarrow e^{+} e^{-}$conversions.

The distribution of mass difference $\Delta m=m(\mu \mu \gamma)-m(\mu \mu)$ is used to distinguish the $\chi_{c 1}$ and $\chi_{c 2}$ states. This distribution is used in place of the three-body invariant mass as some partial cancellation of contributions from the di-muon mass resolution is achieved, resulting in improved overall mass resolution. Non-prompt $\chi_{c}$ candidates produced in the decays of b-hadrons can be experimentally distinguished from prompt $\chi_{c}$ candidates (produced in the primary $p p$ interaction) with the pseudo-proper decay time distribution $\tau$. The pseudo-proper decay time $\tau$ is defined as:

$$
\tau=\frac{L_{x y} \cdot m_{J / \psi}}{p_{T}},
$$

where $m_{J / \psi}$ is the world-average $\mathrm{J} / \psi$ mass, $\mathrm{p}_{\mathrm{T}}$ is the transverse momentum of the $\mathrm{J} / \psi$ candidate and $L_{x y}$ is the distance between the primary $p p$ interaction vertex and the $\mathrm{J} / \psi \rightarrow \mu^{+} \mu^{-}$decay vertex in the transverse plane. A simultaneous fit to the mass difference and pseudo-proper lifetime distributions extracts the $\chi_{c J}$ yields, as shown in Figure 1.

Figure 2 shows the differential cross-sections for prompt $\chi_{c 1}$ and $\chi_{c 2}$ production measured as a function of $\mathrm{p}_{\mathrm{T}}^{J / \psi}$. The measurements are compared with the predictions of next-to-leadingorder (NLO) non-relativistic QCD(NRQCD) $[4,5,6]$, the $\mathrm{k}_{\mathrm{T}}$ factorisation approach $[7,8]$ and 
the leading order (LO) colour-singlet model (CSM) [9] calculations. The NRQCD factorisation approach separates the perturbative production of a heavy quark pair from the non-perturbative evolution of a heavy quark pair into a quarkonium state [3]. NLO NRQCD prediction is in good agreement with data. $k_{T}$ factorisation predicts that the majority of $\mathrm{J} / \psi$ are produced from $\chi_{c}$ at high $\mathrm{p}_{\mathrm{T}}$ and overestimates with respect to data. A significantly lower cross section than data is predicted by LO CSM. Higher order corrections are known to be large and are clearly significant.
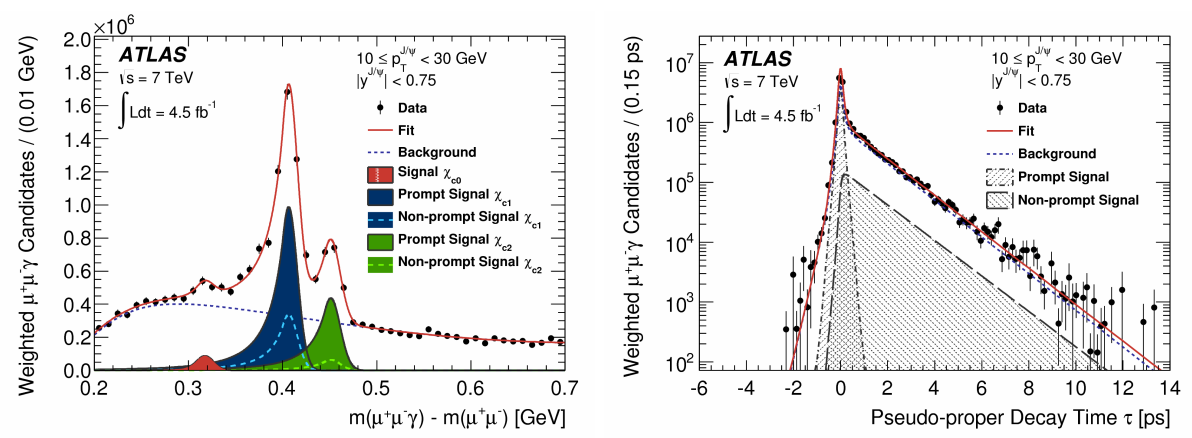

Figure 1: The mass difference $\Delta m=m(\mu \mu \gamma)-m(\mu \mu)$ distribution (left) for $\chi_{c}$ candidates reconstructed within $10 \leq \mathrm{p}_{\mathrm{T}}^{J / \psi}<30 \mathrm{GeV}$ and $\left|\mathrm{y}^{J / \psi}\right|<0.75$. The pseudo-proper lifetime $\tau$ distribution of the same sample of $\chi_{c}$ candidates is also shown (right). The result of the simultaneous fit to both distributions is shown by the overlaid solid red lines. The fitted $\chi_{c J}$ signals are shown by the shaded regions while the fitted background distributions are shown by the dashed blue lines.
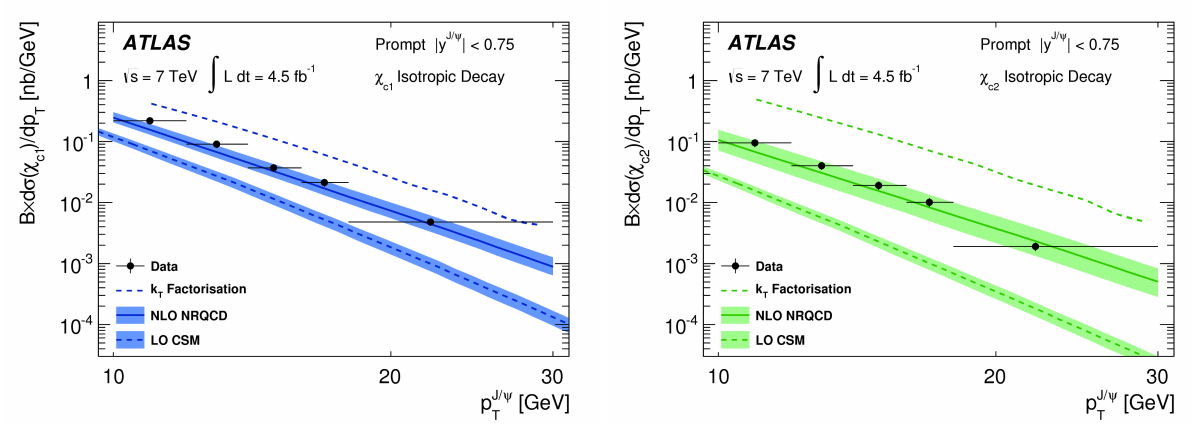

Figure 2: Differential cross sections for prompt $\chi_{c 1}$ (left) and $\chi_{c 2}$ (right) production as a function of $\mathrm{p}_{\mathrm{T}}{ }^{\mathrm{J}}{ }^{\mathrm{T}}$. The predictions of NLO NRQCD, the $\mathrm{k}_{\mathrm{T}}$ factorisation model and the LO CSM are compared to the measurements. The positions of the data points within each bin reflect the average values of the acceptanceand efficiency-corrected $\mathrm{p}_{\mathrm{T}}{ }^{J / \psi}$ distribution of the $\chi_{c}$ candidates within the bin. The error bars represents the total uncertainty on the measurement, assuming unpolarised production. The factor B denotes the product of branching fractions: $\mathrm{B}=B\left(\chi_{c J} \rightarrow J / \psi \gamma\right) \cdot B(\mathrm{~J} / \psi \rightarrow J / \psi \mu \mu)$.

Figure 3 shows the differential cross-sections for non-prompt $\chi_{c 1}$ and $\chi_{c 2}$ production measured as a function of $\mathrm{p}_{\mathrm{T}}^{J / \psi}$ and $\mathrm{p}_{\mathrm{T}} \chi_{c}$. These are the first measurement of non-prompt $\chi_{c}$ production at the LHC. The measurements are generally well described by the fixed order next-to-leading logarithm (FONLL) prediction for $b$-hadron $[10,11]$ production, though the data tend to lie slightly below the predictions at high $\mathrm{p}_{\mathrm{T}}$. Both prompt and non-prompt measurements assume unpolarised production. 

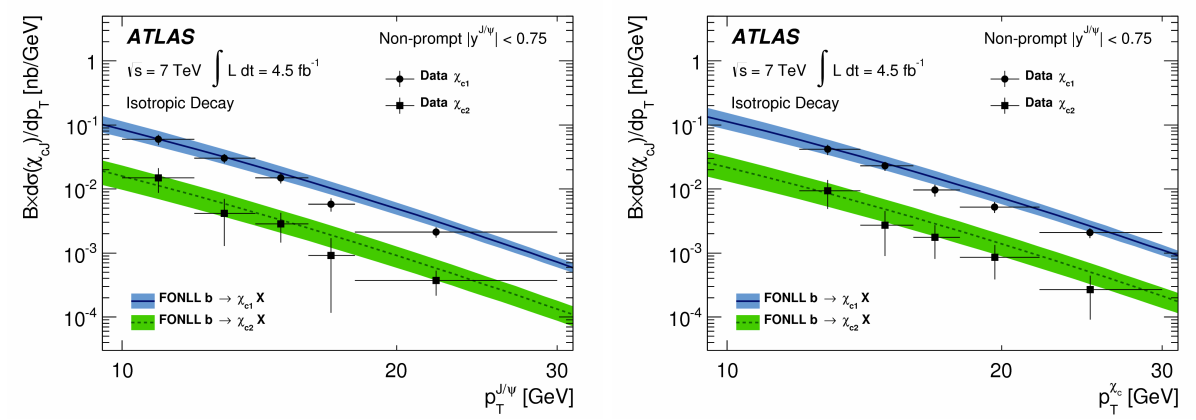

Figure 3: Differential cross sections for non-prompt $\chi_{c 1}$ and $\chi_{c 2}$ production as a function of $\mathrm{p}_{\mathrm{T}}^{J / \psi}$ (left) and $\mathrm{p}_{\mathrm{T}} \chi_{c 1}$ (right). The predictions of FONLL are compared to the measurements. The positions of the data points within each bin reflect the average values of the acceptance- and efficiency-corrected $\mathrm{p}_{\mathrm{T}} \chi_{c 1}$ and $\mathrm{p}_{\mathrm{T}}{ }^{J / \psi}$ distributions of the $\chi_{c}$ candidates within the bin. The error bars represents the total uncertainty on the measurement, assuming unpolarised production. The factor B denotes the product of branching fractions: $\mathrm{B}=B\left(\chi_{c J} \rightarrow J / \psi \gamma\right) \cdot B(\mathrm{~J} / \psi \rightarrow J / \psi \mu \mu)$.
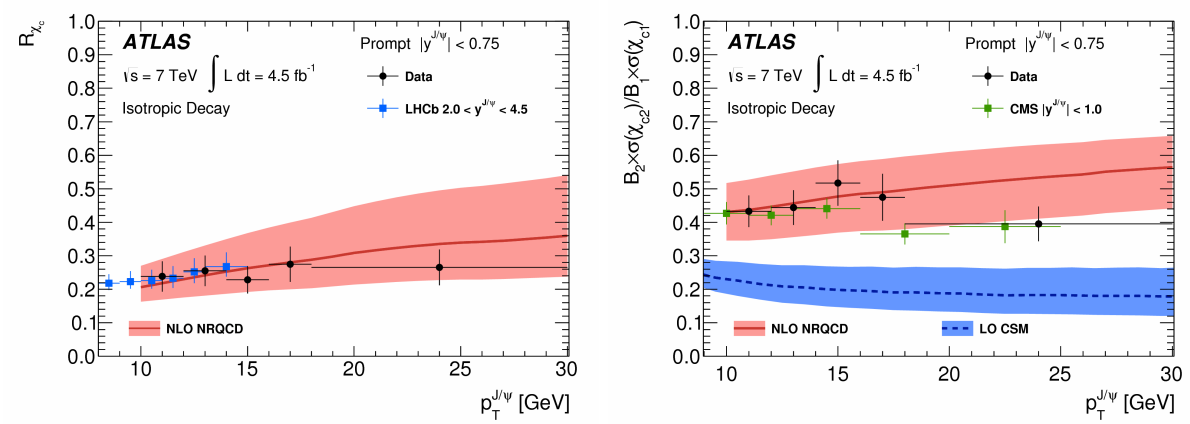

Figure 4: The fraction of prompt $\mathrm{J} / \psi$ produced in $\chi_{c}$ decays as a function of $\mathrm{p}_{\mathrm{T}}^{J / \psi}$ (left). The measurements are compared to the prediction of NLO NRQCD. The measurement from LHCb is also shown. The error bars represents the total uncertainty on the measurement, assuming unpolarised production. The production cross section of prompt $\chi_{c 2}$ relative to prompt $\chi_{c 1}$ measured as a function of $\mathrm{p}_{\mathrm{T}}^{J / \psi}$ (right). The measurements are compared to the predictions of NLO NRQCD and LO CSM. The measurement from CMS is also shown. The error bars represents the total uncertainty on the measurement, assuming unpolarised production. The factors $\mathrm{B} 1$ and $\mathrm{B} 2$ denote the branching fractions $\mathrm{B} 1=B\left(\chi_{c 1} \rightarrow J / \psi \gamma\right)$ and $\mathrm{B} 2=B\left(\chi_{c 2} \rightarrow J / \psi \gamma\right)$ respectively.

The fraction, $R_{\chi_{c}}$, of $\mathrm{J} / \psi$ produced in feed-down from $\chi_{c}$ decays is shown in Figure 4 . The measurements are in good agreement with the NLO NRQCD prediction. LHCb measurements are shown in a more extensive rapidity region. The results show that between $20 \%$ and $30 \%$ of prompt $\mathrm{J} / \psi$ are produced in $\chi_{c}$ feed-down at high $\mathrm{J} / \psi$ transverse momentum.

The prompt cross section ratio is a long standing puzzle. Why are more $\chi_{c 1}$ than $\chi_{c 2}$ produced? The production cross section of prompt $\chi_{c 2}$ relative to prompt $\chi_{c 1}$, measured as a function of $\mathrm{p}_{\mathrm{T}}{ }^{J / \psi}$, is shown in Figure 4. NLO NRQCD is in general agreement with data, probably due to the NLO corrections. LO CSM underestimates the data, which suggests that NRQCD or NLO CSM terms are important. 
The branching fraction $B\left(\mathrm{~B}^{ \pm} \rightarrow \chi_{c 1} \mathrm{~K}^{ \pm}\right)$is measured from

$$
B\left(\mathrm{~B}^{ \pm} \rightarrow \chi_{c 1} K^{ \pm}\right)=A_{B} \cdot \frac{N_{\chi_{c 1}}^{B}}{N_{J / \psi}^{B}} \cdot \frac{B\left(\mathrm{~B}^{ \pm} \rightarrow J / \psi K^{ \pm}\right)}{B\left(\mathrm{~B}^{ \pm} \rightarrow J / \psi \gamma\right)}
$$

using the same dataset and $\chi_{c}$ event selection. Where $A_{B}$ is a factor to correct for the different detector acceptances of the two decays, $N_{\chi_{c 1}}^{B}$ and $N_{J / \psi}^{B}$ are the corrected yields for the signal and reference decay channels. Current world average values are used for the branching fractions. The measured value, $B\left(\mathrm{~B}^{ \pm} \rightarrow \chi_{c 1} \mathrm{~K}^{ \pm}\right)=(4.9 \pm 0.9$ (stat. $) \pm 0.6$ (syst.) $) \times 10^{-4}$, agrees well with the world average as shown in Figure 5.

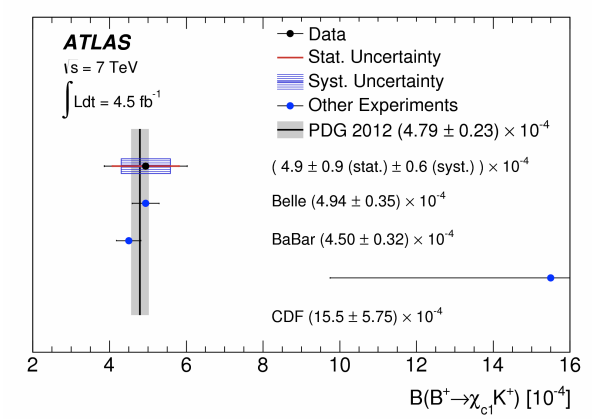

Figure 5: The measurement of the branching fraction $B\left(\mathrm{~B}^{ \pm} \rightarrow \chi_{c 1} \mathrm{~K}^{ \pm}\right)$is compared to the measurements of other experiments and to the PDG world average.

\section{Cross-section measurement of $\psi(2 S) \rightarrow J / \psi\left(\rightarrow \mu^{+} \mu^{-}\right) \pi^{+} \pi^{-}$in $\sqrt{s}=7 \mathrm{TeV}$ pp collisions.}

The prompt and non-prompt production cross-sections for $\psi(2 \mathrm{~S})$ mesons are measured using $2.1 \mathrm{fb}^{-1}$ of $p p$ collision data at a centre-of-mass energy of $7 \mathrm{TeV}$ recorded by the ATLAS experiment at the LHC [12]. The measurement exploits the $\psi(2 S) \rightarrow J / \psi\left(\rightarrow \mu^{+} \mu^{-}\right) \pi^{+} \pi^{-}$decay mode, and probes $\psi(2 \mathrm{~S})$ with transverse momenta in the range $\mathrm{p}_{\mathrm{T}}=10-100 \mathrm{GeV}$ and rapidity $|\mathrm{y}|<2.0$.

Figure 6 shows the measured differential cross-sections for prompt and non-prompt $\psi(2 \mathrm{~S})$ production as a function of $\psi(2 \mathrm{~S})$ transverse momentum for three $\psi(2 \mathrm{~S})$ rapidity intervals.

Theoretical models of prompt $\psi(2 \mathrm{~S})$ production vary significantly in their predictions of overall rate and kinematic dependence. Both the LO and NLO NRQCD predictions describe data reasonably well except for the highest $\mathrm{p}_{\mathrm{T}}$ region, where they overestimate the production rate. In contrast, predictions of the $k_{t}$ factorisation model exhibit a softer $\mathrm{p}_{\mathrm{T}}$ spectrum than observed in the data and clearly undershoot the data in overall rate. Together with the observation of an overestimate of the production rate of $\mathrm{C}$-even $\chi_{c}$ charmonium states in the $k_{t}$ factorisation approach, these measurements provide coherent input to improve this model in the future.

In non-prompt $\psi(2 \mathrm{~S})$ production, both NLO with FONLL fragmentation and FONLL calculations describe the data over a wide range of transverse momentum and across the rapidities probed. Both theoretical approaches overestimate the high $\mathrm{p}_{\mathrm{T}}$ data by a significant factor, despite success in describing the low $\mathrm{p}_{\mathrm{T}}$ spectra. 

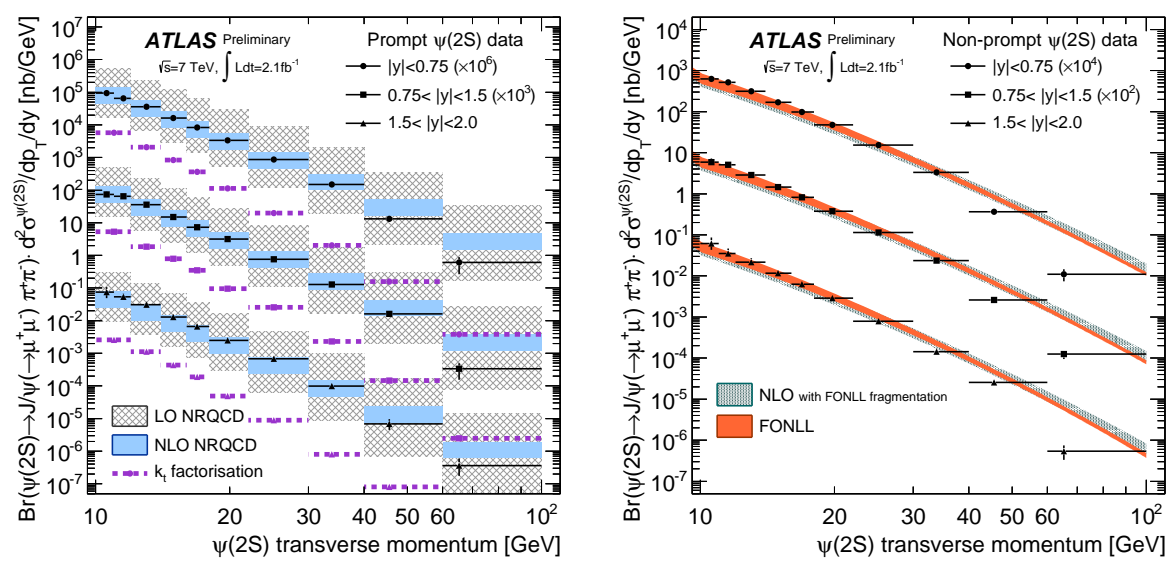

Figure 6: Measured differential cross-sections for prompt (left) and non-prompt (right) $\psi(2 \mathrm{~S})$ production as a function of $\psi(2 \mathrm{~S})$ transverse momentum for three $\psi(2 \mathrm{~S})$ rapidity intervals with comparison to theoretical predictions in the ATLAS fiducial region (left). The data points are at the weighted mean of the $\mathrm{p}_{\mathrm{T}}$ distribution in each $\mathrm{p}_{\mathrm{T}}$ interval, indicated by the horizontal error bars, and the vertical error bars represent the total statistical and systematic uncertainty.

\section{Conclusions.}

The production of heavy quarkonium provides particular insight into QCD theory as its mechanisms of production operate at the boundary of the perturbative and non-perturbative regimes. These measurements provide input towards an improved understanding and theoretical description of QCD.

\section{References}

[1] ATLAS Collaboration, JINST 3(2008)S08003.

[2] ATLAS Collaboration, arXiv:1404.7035.

[3] G. T. Bodwin, E. Braaten, and G. P. Lepage, Phys. Rev. D 51 (1995) 1125.

[4] Y.-Q. Ma, K. Wang, and K.-T. Chao, Phys. Rev. D 83(2011) 111503, arXiv:1002.3987.

[5] H.-S. Shao, Comput. Phys. Commun.184(2013) 2562, arXiv:1212.5293.

[6] Ma, Yan-Qing and Wang, Kai and Chao, Kuang-Ta, Phys. Rev. Lett. 106 (2011) 042002 , arXiv:1009.3655.

[7] S.P. Baranov, A.V. Lipatov, N.P. Zotov, Phys. Rev. D83(2011) 034035, arXiv:1108.2856

[8] S. Baranov, Phys. Rev. D 83(2011)034035.

[9] L.Harland-Lang and W.A. Stirling, http://superchic.hepforge.org/chigen.html.

[10] M. Cacciari et al., JHEP10(2012) 137, arXiv:1205.6344.

[11] M. Cacciari, M. Greco, and P. Nason, JHEP05 (1998) 007, hep-ph/9803400.

[12] ATLAS Collaboration, ATLAS-BPHY-2013-06-001. 